\title{
Association between hOGG1 polymorphism rs1052133 and gastric cancer
}

\author{
Dingding Zhang ${ }^{1, *}$, Xiaoxin Guo ${ }^{1, *}$, Jinliang $\mathrm{Hu}^{2,3, *}$, Guangqun Zeng ${ }^{4}$, Maomin \\ Huang ${ }^{1,5}$, Dandan $\mathbf{Q i}^{1}$, Bo Gong ${ }^{1}$ \\ ${ }^{1}$ Sichuan Provincial Key Laboratory for Disease Gene Study, Hospital of University of Electronic Science and Technology of \\ China and Sichuan Provincial People's Hospital, University of Electronic Science and Technology of China, Chengdu, Sichuan, \\ 610072, China \\ ${ }^{2}$ Institute of Health Policy and Hospital Management, Sichuan Academy of Medical Science and Sichuan Provincial People's \\ Hospital, Chengdu, Sichuan, 610072, China \\ ${ }^{3}$ School of Public Health, Sichuan University, Chengdu, Sichuan, 610041, China \\ ${ }^{4}$ Department of Clinical Laboratory, People's Hospital of Pengzhou, Pengzhou, Sichuan, 611930, China \\ ${ }^{5}$ Department of Immunology, Zunyi Medical College, Zunyi, Guizhou, 563000, China \\ *These authors contributed equally to this study \\ Correspondence to: Bo Gong, email: gongbo2007@hotmail.com
}

Keywords: gastric cancer, meta-analysis, hOGG1 rs1052133, association

Received: January 10, 2017 Accepted: March 04, 2017 Published: March 11, 2017

Copyright: Zhang et al. This is an open-access article distributed under the terms of the Creative Commons Attribution License (CC-BY), which permits unrestricted use, distribution, and reproduction in any medium, provided the original author and source are credited.

\section{ABSTRACT}

Purpose: To conduct a comprehensive evaluation of the association of the human8-oxoguanine glycosylase 1 (hOGG1) gene polymorphism rs1052133 with gastric cancer (GC) through a systematic review and meta-analysis of genetic association study.

Results: A total of 15 articles from published papers were included in our analysis. The meta-analyses for hOGG1 rs1052133, composed of 4024GC patients and 6022 controls, showed low heterogeneity for the included populations in all the genetic models, except for the Caucasian population under allelic genetic model, the Asian population under addictive model and Caucasian population under dominant model. The analyses of all the genetic models in overall pooled populations did not identify any significant association between GC and hOGG1 rs1052133 (Allelic model: C vs. G, $p=0.746$; Addictive model: CC vs. GG, $p=0.888$; Recessive model: CC +GC vs. GG, $p=0.628$; Dominant model: CC vs. GG $+G C, p=0.147$ ), even though stratified analyses were conducted in different ethnicities under each genetic model.

Materials and Methods: All case-control association studies on hOGG1 and GC reported up to December 15, 2016 in PubMed, Embase, Web of Science, and the Chinese Biomedical Database were retrieved. Odds ratios (ORs) and $95 \%$ confidence intervals ( $95 \% \mathrm{CIs}$ ) were calculated for single-nucleotide polymorphism (SNP) using fixed- and random- effects models according to between-study heterogeneity. Publication bias analyses were conducted using Begg test.

Conclusions: This meta-analysis showed there was no association between hOGG1 rs1052133 and GC. Given the limited sample size, further investigations including more ethnic groups are required to validate the association.

\section{INTRODUCTION}

Gastric cancer (GC) is one of the most common cancers and the leading causes of cancer death in the world [1], however, there is a gradually decrease in incidence and mortality rates in most developed countries [2]. GC is a multifactorial disease caused by dietary, genetic and environmental factors [3, 4]. Although the pathogenetic mechanism of GC is very complicated and is still not fully understood, more and more evidence has been shown that there is a correlation between genetic polymorphisms and GC risk [5-7].

To date, many genetic polymorphisms in the carcinogen detoxification, antioxidant protection, DNA 
repair and cell proliferation processes have been reported to play a crucial role in the development of GC [8]. The human 8-oxoguanine glycosylase 1 (hOGG1) gene, specifically involved in the repair of DNA oxidative damage in the base excision response pathway, has been shown to be associated with a variety of cancers [9-13]. As an important component of DNA repair pathway, $h O G G 1$ encodes a DNA glycosylase enzyme that actively removes 8-hydroy-2-deoxyguanine, which is highly mutagenic and a major form of oxidative DNA damage $[14,15]$. The dysfunction of hOGG1 might result in the DNA repair deficiency and then induce gene mutation and cell canceration. Functional studies showed that the hOGG1 variant had normal enzymatic activity, but maintained greater sensitivity to oxidation than wildtype hOGG1 protein [16].

The $h O G G 1$ gene has been regarded as a candidate for involvement in the underlying cause of GC and the hOGG1 polymorphism rs1052133 has been widely evaluated in association with GC across different ethnicities [11, 17-31]. hOGG1 polymorphism rs1052133 has been reported to be associated with an altered risk for GC in Chinese, Japanese and Caucasian populations [11, 18, 19, 32]. However, other reported studies on the association between $h O G G 1$ rs 1052133 and GC risk are inconclusive and conflicting [20-31]. Such inconsistence and heterogeneity could be caused by different sample sizes and diversities in multiple ethnic cohorts. Metaanalysis, which combined all studies with the same criteria, could be helpful to comprehensively explain the association of $h O G G 1$ rs 1052133 with GC and provide some new clues for the research on GC. Therefore, in this study we conduct a systematic review and meta-analysis of all association studies on hOGGl rs1052133 with $\mathrm{GC}$, to summarize and evaluate the association between hOGG1 rs1052133and GC.

\section{RESULTS}

\section{Literature search and characteristics}

A flow diagram (Figure 1) shows the selection process of studies included in our analysis. The initial search strategy yielded 31 articles. Among them, two review articles were excluded because of the publication type and six articles were excluded because of unrelated topics. The full text of the remaining 23 studies was retrieved and reviewed. Eight articles were excluded after full-text review, five studies were not case-control studies and three articles were experimental studies. Finally, 15 articles were identified that met the inclusion criteria and included for the meta-analysis. The analyzed SNP was successfully genotyped and was within HWE (except the genotype in control subjects from the Takezaki's study) across all the included studies. All of the articles were case- control studies and all the cases were histopathologically confirmed as GC. The characteristics of these included articles are listed in Table 1 and Table 2. The NOS results showed that the score ranged from 7 to 8 with an average of 7.50, which indicated that the methodological quality of these selected articles was generally reliable.

\section{Meta-analysis results}

Fifteen studies provided results of the association of hOGG1 rs1052133 with GC and a total of 10046 subjects (4024GC patients and 6022 controls) were tested for $h O G G 1$ rs1052133 in this meta analysis. Stratified analyses were conducted based on three groups, including Asian [11, 18, 24, 26, 27, 29-31], Caucasian $[19,21,22,25]$ and other ethnicities [20, 23, 28], and the heterogeneity of $h O G G 1$ rs1052133 in Asian, Caucasian, other and pooled populations were evaluated firstly. The results showed low heterogeneity for the included populations under all the genetic models by fixed-effect analysis, except for the Caucasian population under allelic genetic model $\left(P=0.052, I^{2}=61.2 \%\right)$, the Asian population under recessive model $\left(P=0.021, I^{2}=57.6 \%\right)$ and Caucasian population under dominant model $(P=$ $0.054, I^{2}=60.7 \%$ ), the random-effect analysis was thus adopted for them under each genetic model (Table 3 ).

Our meta-analysis showed that there was no significant association of $h O G G 1$ rs 1052133 with GC in the overall pooled populations under all the genetic models (Allelic model: C vs. G, OR $=1.016,95 \%$ CI 0.924 to 1.117, $p=0.746 ; I^{2}=40.6$; Addictive model: CC vs. $\mathrm{GG}, \mathrm{OR}=1.010,95 \% \mathrm{CI} 0.883$ to $1.155, p=0.888 ; I^{2}$ $=24.2$; Recessive model: $\mathrm{CC}+\mathrm{GC}$ vs. $\mathrm{GG}, \mathrm{OR}=0.969$, $95 \%$ CI 0.864 to $1.087, p=0.628 ; I^{2}=40.5$; Dominant model: $\mathrm{CC}$ vs. $\mathrm{GG}+\mathrm{GC}, \mathrm{OR}=1.067,95 \% \mathrm{CI} 0.977$ to $1.165, p=0.147 ; I^{2}=4$; Table 3 ). Figure 2 shows the forest plot of estimates of odds ratios of the association of $h O G G 1$ rs 1052133 with GC. When stratified analyses were conducted to explore further association of $h O G G 1$ rs1052133 with GC in different ethnicities under each genetic model, no significant association was detected between hOGG1 rs1052133 and GC, either (Table 3 and Figure 2B-2D).

\section{Publication bias and sensitivity analysis}

Publication biases were assessed by the Egger's test quantitatively and the result for $h O G G 1$ rs1052133 based on the 15 included studies did not observe any obvious evidence of publication bias in the overall analyses under all genetic models (Table 3 and Figure 3). The effect of each study on the pooled OR was also assessed by sequential omission of individual studies. We did not find that the exclusion of any single study alter the significance of the final pooled OR. 
Table 1: Characteristics of the fifteen studies included in this meta-analysis

\begin{tabular}{|c|c|c|c|c|c|c|c|c|c|}
\hline \multirow{2}{*}{ Study } & \multirow{2}{*}{ Country } & \multirow{2}{*}{ Study population } & \multirow{2}{*}{ Ethnicity } & \multicolumn{2}{|c|}{ Study design } & \multirow{2}{*}{$\begin{array}{l}\text { Genotyping } \\
\text { method }\end{array}$} & \multicolumn{2}{|c|}{ Mean Age } & \multirow{2}{*}{ NOS } \\
\hline & & & & Case & Control & & Case & Control & \\
\hline Lu, et al. 2016 & China & Chinese & Asian & $\mathrm{CB}$ & HB & SNaPshot & $63.1 \pm 10.7$ & $63.3 \pm 11.0$ & 8 \\
\hline $\mathrm{Hu}$, et al. 2015 & China & Chinese & Asian & HB & $\mathrm{HB}$ & PCR-LDR & - & - & 8 \\
\hline Engin, et al. 2011 & Turkey & Turkish & Others & $\mathrm{HB}$ & $\mathrm{HB}$ & PCR-RFLP & $60.4 \pm 1.3$ & $55.5 \pm 1.3$ & 7 \\
\hline Liu, et al. 2011 & China & Chinese & Asian & HB & $\mathrm{CB}$ & PCR-HMR & $60.2 \pm 10.4$ & $59.3 \pm 11.8$ & 8 \\
\hline Canbay, et al. 2010 & Turkey & Turkish & Others & $\mathrm{CB}$ & PB & PCR-RFLP & $60.1 \pm 20.9$ & $52.8 \pm 27.2$ & 8 \\
\hline Sun, et al. 2010 & China & Chinese & Asian & $\mathrm{HB}$ & PB & PCR-RFLP & $59.6 \pm 11.2$ & $43.6 \pm 10.3$ & 7 \\
\hline Malik, et al. 2010 & India & Indian & Asian & $\mathrm{CB}$ & PB & PCR-SSCP & $55.9 \pm 9.7$ & $58.0 \pm 12.7$ & 7 \\
\hline Palli, et al. 2010 & Italy & Italian & Caucasian & $\mathrm{CB}$ & PB & Taqman & $68.8 \pm 9.9$ & $55.5 \pm 7.0$ & 7 \\
\hline Farinati, et al. 2008 & Italy & Italian & Caucasian & $\mathrm{CB}$ & PB & PCR-RFLP & 68 & 46 & 8 \\
\hline Capella, et al. 2008 & Spain & Spanish & Caucasian & $\mathrm{CB}$ & PB & Probe & $50.5 \pm 20.5$ & $50.5 \pm 20.5$ & 8 \\
\hline Poplawskiet al. 2006 & Poland & Polish & Caucasian & $\mathrm{HB}$ & PB & PCR-SSCP & $62.4 \pm 27.4$ & $62.4 \pm 27.4$ & 8 \\
\hline Tsukino, et al. 2004 & Japan & Japanese & Asian & $\mathrm{HB}$ & HB & PCR-SSCP & $57.5 \pm 9.5$ & $57.1 \pm 9.5$ & 7 \\
\hline Takezaki, et al. 2002 & China & Chinese & Asian & $\mathrm{CB}$ & PB & PCR- SSCP & $65 \pm 15$ & $65 \pm 14$ & 7 \\
\hline Hanaoka, et al. 2001 & Japan & Japanese Brazilian & Others & HB & HB & PCR-SSCP & $65 \pm 13$ & $65 \pm 12$ & 7 \\
\hline Hanaoka, et al. 2001 & Japan & $\begin{array}{c}\text { non-Japanese } \\
\text { Brazilian }\end{array}$ & Others & $\mathrm{HB}$ & HB & PCR-SSCP & $59 \pm 8$ & $58 \pm 8$ & 8 \\
\hline Shinmura, et al. 1998 & Japan & Japanese & Asian & $\mathrm{HB}$ & $\mathrm{PB}$ & PCR-SSCP & - & - & 7 \\
\hline
\end{tabular}

PB, population based; CB, clinic or institute based; HB, hospital based; NOS, Newcastle-Ottawa Scale; -Data unavailable.

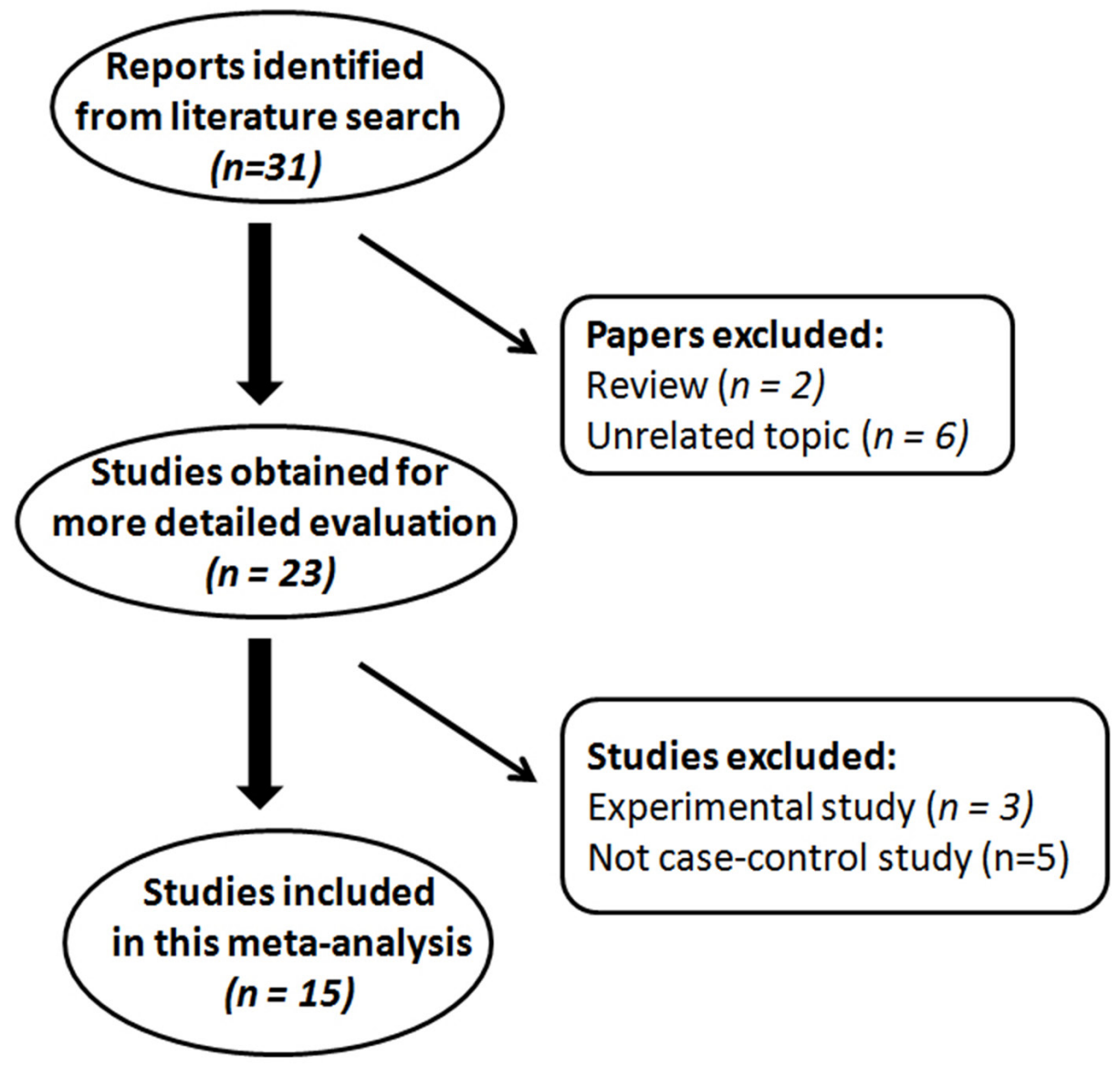

Figure 1: Flow diagram of literature search and study selection for meta-analysis. 
Table 2: Genotype frequencies of hOGG1 rs1052133 among gastric cancer cases and controls in the included studies

\begin{tabular}{|c|c|c|c|c|c|c|c|c|c|c|c|c|}
\hline \multirow[t]{2}{*}{ Study } & \multicolumn{2}{|c|}{ Sample size } & \multicolumn{3}{|c|}{$\begin{array}{l}\text { Genotypes } \\
\text { (Case) }\end{array}$} & \multicolumn{3}{|c|}{$\begin{array}{l}\text { Genotypes } \\
\text { (Control) }\end{array}$} & \multicolumn{2}{|c|}{$\begin{array}{c}\text { Allele } \\
\text { Frequency (G) }\end{array}$} & \multirow{2}{*}{$\begin{array}{c}\text { OR, 95\%CI } \\
\text { (Allellc Model) }\end{array}$} & \multirow[t]{2}{*}{$P$} \\
\hline & Case & Control & $\mathrm{CC}$ & CG & GG & $\mathrm{CC}$ & CG & GG & Case & Control & & \\
\hline Lu, et al. 2016 & 1279 & 1434 & 477 & 591 & 211 & 525 & 702 & 207 & 0.396 & 0.389 & $0.97(0.87-1.08)$ & 0.604 \\
\hline Hu, et al. 2015 & 436 & 372 & 154 & 210 & 72 & 128 & 176 & 68 & 0.406 & 0.419 & $1.06(0.87-1.29)$ & 0.586 \\
\hline $\begin{array}{l}\text { Engin, et al. } \\
2011\end{array}$ & 106 & 116 & 53 & 42 & 11 & 51 & 47 & 18 & 0.302 & 0.358 & $1.29(0.87-1.92)$ & 0.211 \\
\hline Liu, et al. 2011 & 618 & 913 & 114 & 302 & 202 & 144 & 447 & 322 & 0.571 & 0.594 & $1.10(0.95-1.27)$ & 0.199 \\
\hline $\begin{array}{l}\text { Canbay, et al. } \\
2010\end{array}$ & 40 & 247 & 24 & 13 & 3 & 171 & 69 & 7 & 0.238 & 0.168 & $0.65(0.37-1.14)$ & 0.131 \\
\hline Sun, et al. 2010 & 73 & 255 & 21 & 19 & 33 & 72 & 119 & 64 & 0.582 & 0.484 & $0.67(0.46-0.98)$ & 0.037 \\
\hline $\begin{array}{l}\text { Malik, et al. } \\
2010\end{array}$ & 108 & 195 & 50 & 51 & 7 & 94 & 89 & 12 & 0.301 & 0.290 & $0.95(0.66-1.36)$ & 0.772 \\
\hline Palli, et al. 2010 & 304 & 545 & 192 & 101 & 11 & 325 & 191 & 29 & 0.202 & 0.228 & $1.17(0.92-1.49)$ & 0.212 \\
\hline $\begin{array}{l}\text { Farinati, et al. } \\
2008\end{array}$ & 50 & 43 & 33 & 15 & 2 & 36 & 7 & 0 & 0.190 & 0.081 & $0.38(0.15-0.95)$ & 0.033 \\
\hline $\begin{array}{l}\text { Capella, et al. } \\
2008\end{array}$ & 438 & 1026 & 279 & 137 & 22 & 621 & 352 & 53 & 0.207 & 0.223 & $1.10(0.91-1.34)$ & 0.320 \\
\hline $\begin{array}{l}\text { Poplawski, et al. } \\
2006\end{array}$ & 28 & 33 & 22 & 6 & 0 & 18 & 15 & 0 & 0.107 & 0.227 & $2.45(0.88-6.82)$ & 0.079 \\
\hline Tsukino, 2004 & 142 & 271 & 32 & 75 & 35 & 74 & 141 & 56 & 0.511 & 0.467 & $0.84(0.63-1.12)$ & 0.232 \\
\hline Takezaki, 2002 & 101 & 198 & 20 & 61 & 20 & 30 & 120 & 48 & 0.500 & 0.545 & $1.20(0.85-1.68)$ & 0.292 \\
\hline Hanaoka, 2001 & 58 & 127 & 20 & 29 & 9 & 44 & 56 & 27 & 0.405 & 0.433 & $1.12(0.72-1.75)$ & 0.614 \\
\hline Hanaoka, 2001 & 208 & 205 & 133 & 67 & 8 & 123 & 74 & 8 & 0.200 & 0.220 & $1.13(0.81-1.58)$ & 0.480 \\
\hline Shinmura, 1998 & 35 & 42 & 9 & 16 & 10 & 15 & 20 & 7 & 0.514 & 0.405 & $0.64(0.34-1.22)$ & 0.174 \\
\hline
\end{tabular}

MAF, minor allele frequency; OR, odds ratio; CI, confidence intervals; $\mathrm{P}, p$ value for $Z$ test.

\section{DISCUSSION}

As a powerful statistical method, meta-analysis could provide a quantitative approach for pooling the variant results on the same topic to estimate and explain their diversity. This led us to conduct this meta-analysis of 15 published case-control studies, which may help us in distinguishing the truth from the false, and to explore a more robust estimate of the effect of $h O G G 1$ rs 1052133 on GC. The hOGG1 rs1052133 has been thought to constitute a candidate genetic risk factor for GC [11, 18, 19, 32]; however, several other studies have investigated the genetic effect of hOGG1 rs1052133 on GC susceptibility with conflicting results [20-31]. In this study, our metaanalysis showed there was no association between $h O G G 1$ rs 1052133 and GC in all genetic models, suggesting that hOGG1 rs1052133 may not affect individual susceptibility to GC.

GC is considered to be a common, complex and multifactorial disease, and is estimated to have a significant heritable component. GC ranks as the third leading cause of cancer mortality worldwide and confers a 5 -year survival of $20 \%$ [33]. Identifying causative genes has helped to understand the disease process and may aid in prevention. About $1 \%-3 \%$ of GC could be attributed to inherited cancer predisposition syndromes. More advances have been made in human GC genetics, but there is still to be known [8]. To date, Germline E-cadherin/ $\mathrm{CDH} 1$ mutations have been identified in families with an autosomal dominant inherited predisposition to diffuse GC [34]. hOGG1 has been reported to be related to GC risk in several studies [11, 18, 19, 32]. It is an important glycosylase enzyme that plays a critical role in the repair of DNA oxidative damage $[14,15]$. The dysfunction of hOGG1 might lead to deficiency of DNA repair and result in cell canceration. hOGG1 variant has been shown to exhibit lower DNA repair activity and greater sensitivity to oxidation than wildtype hOGG1 protein [16].

The association between $h O G G 1$ rs 1052133 and GC in previous studies were inconsistent. hOGG1 rs1052133 was reported to be significantly associated with GC in Japanese and Chinese patients with GC [11, 18, 19, 32]. However, no evidence of association between hOGG1 rs1052133 and GC was detected in Turkish, Spanish, 
Italian, Polish and Indian studies [21-25, 28], meanwhile, the association of hOGG1 rs1052133 with GC was not replicated in other Japanese and Chinese populations $[20,26,27]$. Recently, the association between hOGG1 rs1052133 with GC risks was also accessed in Lu et al' study with 1,275 GC patients and 1,436 controls [29] and in Hu et al's study with $2745 \mathrm{GC}$ patients and 4588 controls [30], respectively. Both the two studies demonstrated that the significant association was not present between $h O G G 1$ rs1052133 and GC. In order to provide powerful statistical analysis, we conduct this study with more samples (a total of 4024GC patients and 6022 controls) to evaluate the association between hOGG1 rs1052133 and GC. Our results did not show any significant association of hOGG1 rs1052133 with GC, even across different ethnic populations. Therefore, our analyses failed to conclude whether hOGG1 rs 1052133 is really a GC-associated SNP and more replication data is needed to validate this association. Of course, other factors, such as environment and different lifestyle, might play roles in these differences as well. Further analysis should be performed in more large-scale cohorts or case-control studies to explore the association of $h O G G 1$ rs 1052133 with GC. Future studies on gene-environment interaction should also be considered.
This systematic review and meta-analysis has sufficient power to evaluate and review all the published genetics studies in hOGG1 rs1052133, however, some potential limitations of our study on the understanding of $h O G G 1$ in GC genetics should be considered. (1) This meta-analysis was mainly based on the studies with 2950 GC patients and 3817 controls in Japanese and Chinese populations [11, 18, 20, 26, 27, 29-31]. Besides, only 1074 GC patients and 2205 controls in Turkish, Indian, Italian, Spanish and Polish populations were included in the present study. It may restrict our conclusions which indicate the need for larger sample sizes in other ethnic populations. (2) There was a high heterogeneity detected in the Caucasian population under allelic genetic model, in the Asian population under addictive model and in the Caucasian population under dominant model. It is possible that different populations with different clinical patient characteristics were included. (3) GC is a multifactorial disease that results from complex interactions between various genetic factors and other factors. Therefore, our results may be influenced by confronting factors, such as age, gender, environment, and lifestyle. If the investigation of gene-environment interactions in different ethnic subgroups could be carried out, we might get

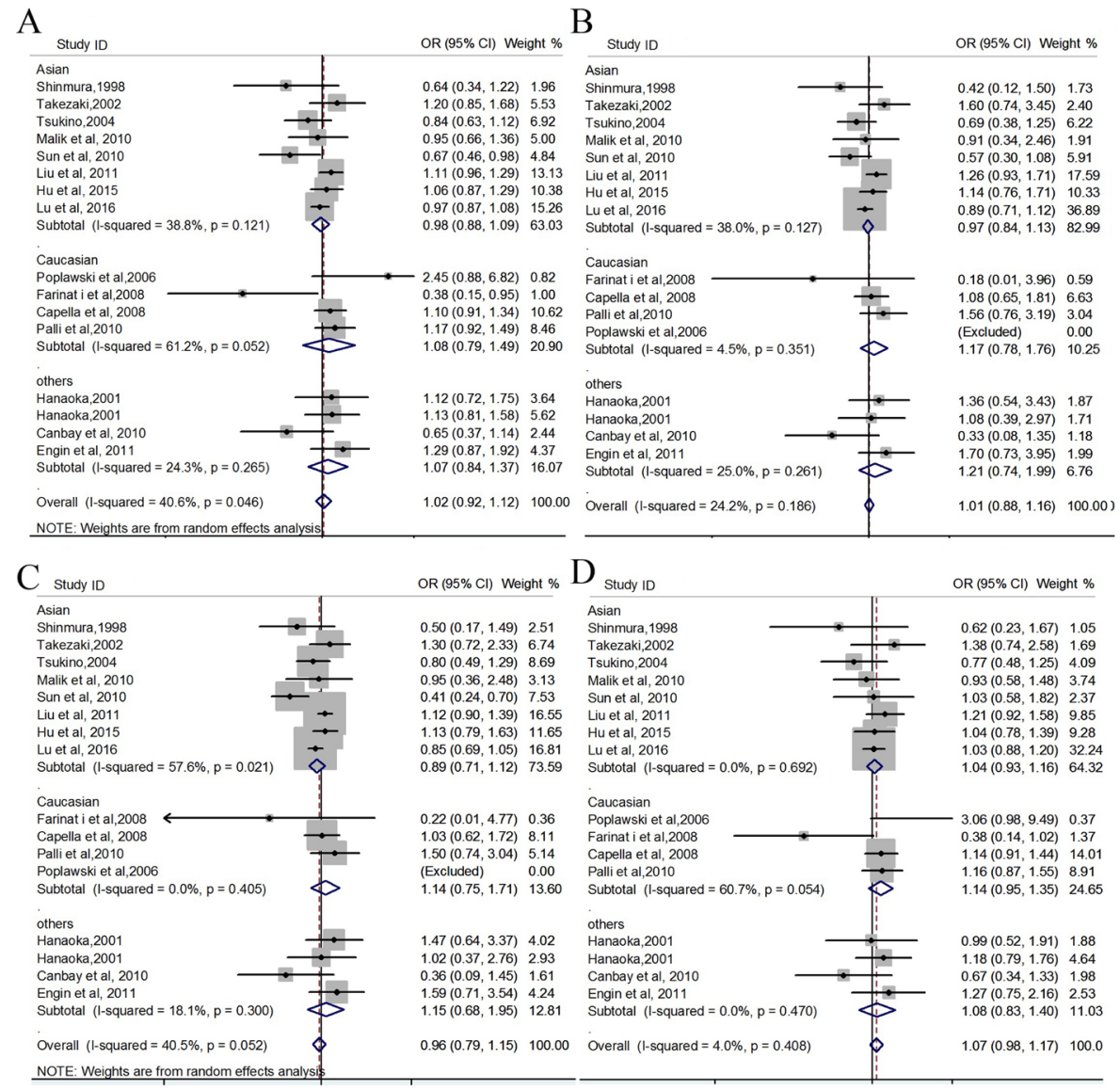

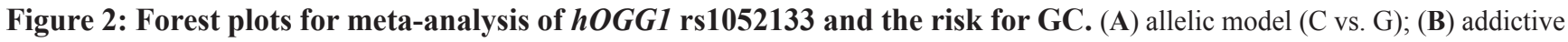
genetic model (CC vs. GG); (C) recessive genetic model (CC + GC vs. GG); (D) dominant genetic model (CC vs. GG+GC). 
Table 3: Pooled measure for the association of $h O G G 1$ rs1052133 and gastric cancer different allelic models

\begin{tabular}{|c|c|c|c|c|c|c|c|}
\hline \multirow{2}{*}{ Genetic model } & \multirow{2}{*}{ Ethnicity } & \multirow{2}{*}{ Pooled OR( 95\% CI) } & \multicolumn{2}{|c|}{ Heterogeneity } & \multicolumn{2}{|c|}{ Test for overall effect } & \multirow{2}{*}{$\frac{\text { Begg test }}{P \Delta}$} \\
\hline & & & $P$ & $I^{2}(\%)$ & $Z$ & $P$ & \\
\hline \multirow{4}{*}{$\begin{array}{l}\text { Allelic model } \\
\text { ( C vs. G) }\end{array}$} & Asian & $0.979(0.879-1.091)$ & 0.121 & 38.8 & 0.38 & 0.705 & \multirow{4}{*}{0.344} \\
\hline & Caucasian $\Delta$ & $1.085(0.789-1.492)$ & 0.052 & 61.2 & 0.50 & 0.616 & \\
\hline & others & $1.072(0.841-1.366)$ & 0.265 & 24.4 & 0.56 & 0.574 & \\
\hline & Overall & $1.016(0.924-1.117)$ & 0.046 & 40.6 & 0.32 & 0.746 & \\
\hline \multirow{4}{*}{$\begin{array}{l}\text { Addictive model } \\
\text { (CC vs. GG) }\end{array}$} & Asian & $0.973(0.839-1.130)$ & 0.127 & 38.0 & 0.35 & 0.723 & \multirow{4}{*}{0.235} \\
\hline & Caucasian & $1.171(0.778-1.764)$ & 0.351 & 4.5 & 0.76 & 0.450 & \\
\hline & others & $1.210(0.736-1.989)$ & 0.261 & 25.0 & 0.75 & 0.452 & \\
\hline & Overall & $1.010(0.883-1.155)$ & 0.186 & 24.2 & 0.14 & 0.888 & \\
\hline \multirow{4}{*}{$\begin{array}{l}\text { Recessive model } \\
(\mathrm{CC}+\mathrm{GC} \text { vs. GG })\end{array}$} & Asian $\Delta$ & $0.939(0.830-1.064)$ & 0.021 & 57.6 & 1.01 & 0.313 & \multirow{4}{*}{0.322} \\
\hline & Caucasian & $1.128(0.752-1.692)$ & 0.405 & 0.0 & 0.61 & 0.543 & \\
\hline & others & $1.223(0.765-1.956)$ & 0.300 & 18.1 & 0.53 & 0.594 & \\
\hline & Overall & $0.969(0.864-1.087)$ & 0.052 & 40.5 & 0.48 & 0.628 & \\
\hline \multirow{4}{*}{$\begin{array}{l}\text { Dominant model } \\
\text { (CC vs. GG +GC) }\end{array}$} & Asian & $1.039(0.930-1.160)$ & 0.692 & 0.0 & 0.68 & 0.498 & \multirow{4}{*}{0.260} \\
\hline & Caucasian $\Delta$ & $1.136(0.954-1.353)$ & 0.054 & 60.7 & 1.43 & 0.152 & \\
\hline & others & $1.079(0.828-1.404)$ & 0.470 & 0.0 & 0.56 & 0.575 & \\
\hline & Overall & $1.067(0.977-1.165)$ & 0.408 & 4.0 & 1.45 & 0.147 & \\
\hline
\end{tabular}

*OR, odds ratio; CI, confidence intervals; $\mathrm{P}, p$ value for $Z$ test; $I^{2}(\%)$, the value to identify heterogeneity. $\Delta$ Pooling model is random effect (Inverse Variance heterogeneity).

$\Delta$ Continuity corrected.

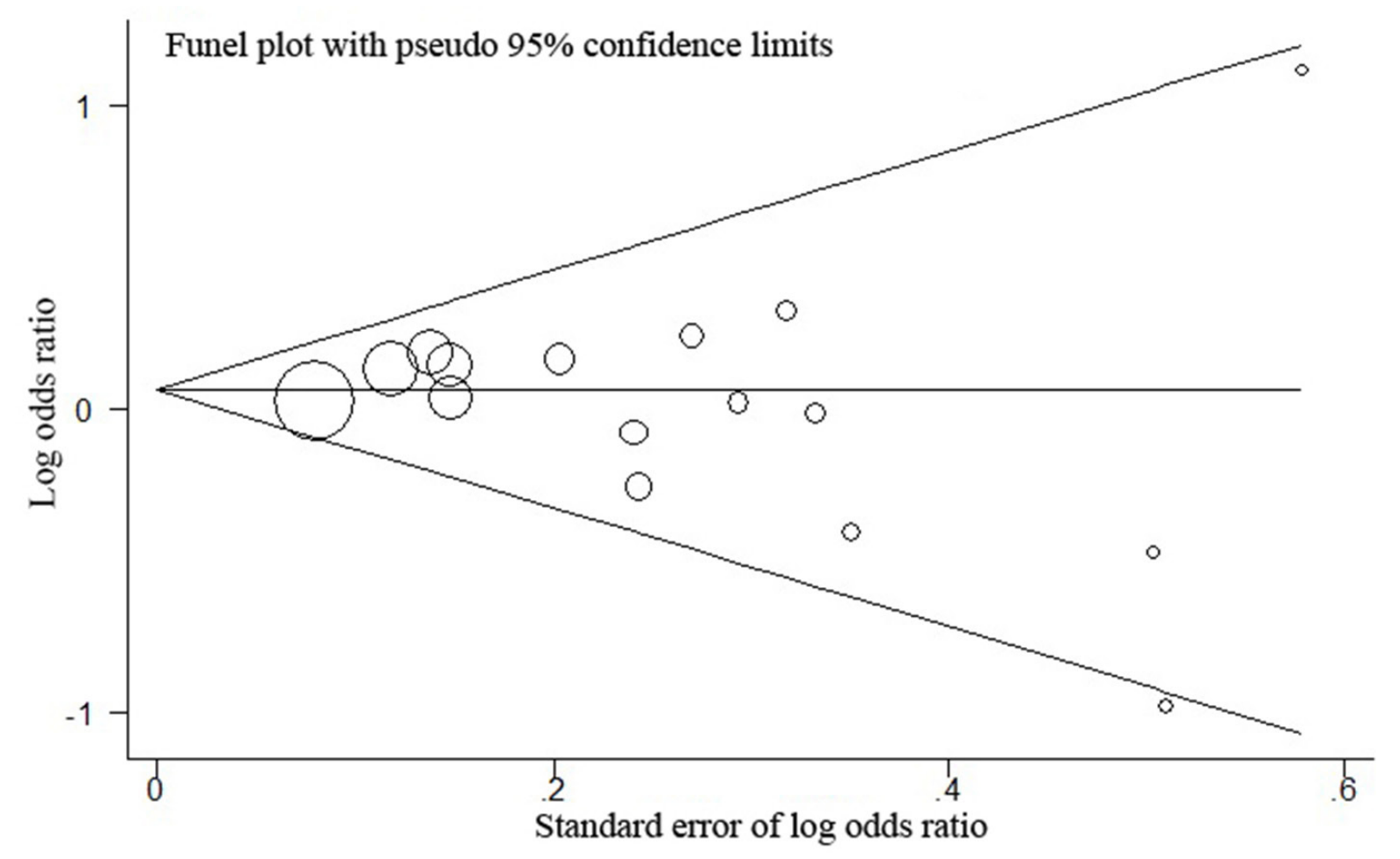

Figure 3: Funnel plot analysis for publication bias between $\boldsymbol{h O G G}$ rs1052133 and GC risk. P for publication bias of this funnel plot for dominant model (CCvs.GG+GC) with all 15 studies is 0.260 . 
more conclusive claims about the association of $h O G G 1$ rs1052133 with GC.

In conclusion, this meta-analysis showed that hOGG1 rs1052133 is not associated with GC under all the genetic models, suggesting that it may not affect individual susceptibility to GC. Further investigations with larger sample sizes and more ethnic groups are required to validate the association and confirm the roles of $h O G G 1$ in GC.

\section{MATERIALS AND METHODS}

\section{Searching strategy}

A systematic literature search using the databases PubMed, Embase, Web of Science, and the Chinese Biomedical Database was conducted, to identify all published studies on the association of hOGG1 polymorphisms with GC from their starting date to December 15, 2016. Following keywords were used: "cancer," "gastric cancer," "human 8-oxoguanine glycosylase," "hOGG1," “'rs1052133," "polymorphism(s)," "variant(s)," and "mutation(s)." The internet was searched using the Google search engine. Reference lists of the retrieved articles and reviews were manually checked for additional articles, to identify studies not yet included in the electronic searches.

\section{Inclusion and exclusion criteria}

The eligible articles were considered if they (1) evaluated associations between $h O G G 1$ rs1052133 and GC; (2) used a case-control design to compare GC cases and normal controls in defined populations; (3) gave an OR with $95 \%$ CI or other available data from which they could be estimated; and (4) were original research articles, not reviews or comments. Excluded were abstracts from conferences, full texts without raw data available for retrieval, republished data, duplicate studies and reviews.

\section{Data extraction}

Two observers (DDZ and XNG) independently abstracted data from all eligible publications onto paper data collection forms. Two reviewers (JLH and GQZ) were blinded to the details (title, author, and academic address) of these studies during assessment. Disagreements were resolved by discussion until a consensus was achieved. Otherwise, a third investigator was consulted to resolve the dispute. The following items were collected from each study: first author's surname, year of publication, statistical data, ethnicity of subjects, whether HardyWeinberg equilibrium (HWE) was examined in controls, genotyping method, total numbers of cases and controls, as well as total numbers of cases and controls for each $h O G G 1$ genotypes, respectively.

\section{Quality assessment}

The Newcastle-Ottawa Scale (NOS) was used to assess the quality of the included studies by two investigators independently. Through the rating system, the NOS was conducted to judge the study quality based on three aspects, which were selection, comparability, and exposure situation in case-control studies. Rating scores range from 0 (worst) to 9 (best). Studies with a score of 7 or greater were thought to have an adequate or good quality.

\section{Statistical analysis}

A pooled OR with its corresponding 95\% CI was used as a measure of the association of hOGG1 rs1052133 with GC. For genotypic comparison, allelic, addictive, dominant and recessive models were applied in the investigation of the disease association. We conducted stratified analyses by ethnicity, including Asian populations (Japanese, Chinese and Indian), Caucasian populations (Polish, Italian and Spanish), other populations (Turkish, Japanese Brazilian and non-Japanese Brazilian) and pooled populations. Sensitivity analysis was carried out by excluding one study at a time to explore whether the results were influenced by a specific study. Heterogeneity (between-study inconsistency) was investigated and measured using $I^{2}$ statistic. A $p$ value of $I^{2}<50 \%$ indicated an absence of heterogeneity among studies, the fixedeffect model (Mantel-Haenszel method) was thus used to calculate the pooled ORs. In contrast, if the $p$ value for heterogeneity was $I^{2} \geq 50 \%$, indicating a high degree of heterogeneity between studies, then the random-effect model (DerSimonian-Laird method) was used to evaluate the summary ORs. Begg liner regression test was used to assess the potential publication bias, where a value of $p<0.05$ was considered statistically significant. The Hardy-Weinberg equilibrium (HWE) for each SNP was tested by the $\chi^{2}$ test. All statistical analyses were performed by the Review Manager software (RevMan, version 5.2; The Nordic Cochrane Centre, The Cochrane Collaboration, Copenhagen;2012), and the STATA software (version 12.0, STATA Corp., College Station, TX, USA), as well as the Hardy Weinberg package (version 1.3) in R language (version 2.15.0, http://cran.r-project.org/). Two-sided $P$ values $<0.05$ were considered statistically significant.

\section{Authors' contributions}

Bo Gong conceived and designed the study; Xiaoxin Guo, Guangqun Zeng, Dingding Zhang, Maomin Huang and Dandan Qi collected the data; Jinliang $\mathrm{Hu}$ analyzed the data; Bo Gong and Dingding Zhang contributed to the writing of the manuscript. 


\section{ACKNOWLEDGMENTS}

Supported in part by grants from the Natural Science Foundation of China (81670853(B.G.), 81371048 (B.G.), 81470067 (D.Z.) and 81271049 (D.Z.)); grants from Department of Science and Technology of Sichuan Province (2016SZ0022(D.Z.)); grants from health program of Sichuan(2016213(D.Z.)).

\section{CONFLICTS OF INTEREST}

The authors report no conflicts of interest. The authors alone are responsible for the content and writing of the paper.

\section{REFERENCES}

1. Jemal A, Siegel R, Ward E, Hao Y, Xu J, Thun MJ. Cancer statistics, 2009. CA Cancer J Clin. 2009; 59:225-249.

2. Parkin DM, Bray F, Ferlay J, Pisani P. Global cancer statistics, 2002. CA Cancer J Clin. 2005; 55:74-108.

3. Gonzalez CA, Sala N, Capella G. Genetic susceptibility and gastric cancer risk. Int J Cancer. 2002; 100:249-260.

4. Masuda G, Tokunaga A, Shirakawa T, Togashi A, Kiyama T, Kato S, Matsukura N, Bou H, Watanabe M, Tajiri T. Helicobacter pylori infection, but not genetic polymorphism of CYP2E1, is highly prevalent in gastric cancer patients younger than 40 years. Gastric Cancer. 2007; 10:98-103.

5. Gu H, Yang L, Tang N, Zhou B, Zhu H, Sun Q, Cong R, Wang B. Association of endothelin-converting enzyme-1b C-338A polymorphism with gastric cancer risk: a casecontrol study. Eur J Cancer. 2008; 44:1253-1258.

6. Qian F, Sun BL, Zhang WY, Ke J, Zhu J. Gly82Ser polymorphism of the receptor for advanced glycation end-product (RAGE) potential high risk in patients with colorectal cancer. Tumour Biol. 2014; 35:3171-3175.

7. Yang L, Liu D, Liang S, Guo R, Zhang Z, Xu H, Yang C, Zhu Y. Janus kinase 2 polymorphisms are associated with risk in patients with gastric cancer in a Chinese population. PLoS One. 2013; 8:e64628.

8. Petrovchich I, Ford JM. Genetic predisposition to gastric cancer. Semin Oncol. 2016; 43:554-559.

9. Zhang J, Dhakal IB, Greene G, Lang NP, Kadlubar FF. Polymorphisms in hOGG1 and XRCC1 and risk of prostate cancer: effects modified by plasma antioxidants. Urology. 2010; 75:779-785.

10. Weiss JM, Goode EL, Ladiges WC, Ulrich CM. Polymorphic variation in hOGG1 and risk of cancer: a review of the functional and epidemiologic literature. Mol Carcinog. 2005; 42:127-141.

11. Tsukino H, Hanaoka T, Otani T, Iwasaki M, Kobayashi M, Hara M, Natsukawa S, Shaura K, Koizumi Y, Kasuga Y, Tsugane S. hOGG1 Ser326Cys polymorphism, interaction with environmental exposures, and gastric cancer risk in Japanese populations. Cancer Sci. 2004; 95:977-983.
12. Okasaka T, Matsuo K, Suzuki T, Ito H, Hosono S, Kawase T, Watanabe M, Yatabe Y, Hida T, Mitsudomi T, Tanaka H, Yokoi K, Tajima K. hOGG1 Ser326Cys polymorphism and risk of lung cancer by histological type. J Hum Genet. 2009; 54:739-745.

13. Wang W, Dang S, Li Y, Sun M, Jia X, Wang R, Liu J. hOGG1 Ser326Cys polymorphism and risk of hepatocellular carcinoma among East Asians: a metaanalysis. PLoS One. 2013; 8:e60178.

14. Collins AR, Gaivao I. DNA base excision repair as a biomarker in molecular epidemiology studies. Mol Aspects Med. 2007; 28:307-322.

15. Boiteux S, Radicella JP. The human OGG1 gene: structure, functions, and its implication in the process of carcinogenesis. Arch Biochem Biophys. 2000; 377:1-8.

16. Kohno T, Shinmura K, Tosaka M, Tani M, Kim SR, Sugimura H, Nohmi T, Kasai H, Yokota J. Genetic polymorphisms and alternative splicing of the hOGG1 gene, that is involved in the repair of 8-hydroxyguanine in damaged DNA. Oncogene. 1998; 16:3219-3225.

17. Farinati F, Cardin R, Degan P, Rugge M, Mario FD, Bonvicini P, Naccarato R. Oxidative DNA damage accumulation in gastric carcinogenesis. Gut. 1998; 42:351-356.

18. Takezaki T, Gao CM, Wu JZ, Li ZY, Wang JD, Ding JH, Liu YT, Hu X, Xu TL, Tajima K, Sugimura H. hOGG1 Ser(326)Cys polymorphism and modification by environmental factors of stomach cancer risk in Chinese. Int J Cancer. 2002; 99:624-627.

19. Farinati F, Cardin R, Bortolami M, Nitti D, Basso D, de Bernard M, Cassaro M, Sergio A, Rugge M. Oxidative DNA damage in gastric cancer: CagA status and OGG1 gene polymorphism. Int J Cancer. 2008; 123:51-55.

20. Hanaoka T, Sugimura H, Nagura K, Ihara M, Li XJ, Hamada GS, Nishimoto I, Kowalski LP, Yokota J, Tsugane S. hOGG1 exon7 polymorphism and gastric cancer in case-control studies of Japanese Brazilians and nonJapanese Brazilians. Cancer Lett. 2001; 170:53-61.

21. Poplawski T, Arabski M, Kozirowska D, BlasinskaMorawiec M, Morawiec Z, Morawiec-Bajda A, Klupinska G, Jeziorski A, Chojnacki J, Blasiak J. DNA damage and repair in gastric cancer--a correlation with the hOGG1 and RAD51 genes polymorphisms. Mutat Res. 2006; 601:83-91.

22. Capella G, Pera G, Sala N, Agudo A, Rico F, Del Giudicce G, Plebani M, Palli D, Boeing H, Bueno-deMesquita HB, Carneiro F, Berrino F, Vineis $\mathrm{P}$, et al. DNA repair polymorphisms and the risk of stomach adenocarcinoma and severe chronic gastritis in the EPICEURGAST study. Int J Epidemiol. 2008; 37:1316-1325.

23. Canbay E, Agachan B, Gulluoglu M, Isbir T, Balik E, Yamaner S, Bulut T, Cacina C, Eraltan IY, Yilmaz A, Bugra D. Possible associations of APE1 polymorphism with susceptibility and HOGG1 polymorphism with prognosis in gastric cancer. Anticancer Res. 2010; 30:1359-1364. 
24. Malik MA, Zargar SA, Mittal B. Lack of influence of DNA repair gene OGG1 codon 326 polymorphisms of gastric cancer risk in the Kashmir valley. Asian Pac J Cancer Prev. 2010; 11:165-168.

25. Palli D, Polidoro S, D'Errico M, Saieva C, Guarrera S, Calcagnile AS, Sera F, Allione A, Gemma S, Zanna I, Filomena A, Testai E, Caini S, et al. Polymorphic DNA repair and metabolic genes: a multigenic study on gastric cancer. Mutagenesis. 2010; 25:569-575.

26. Sun LM, Shang Y, Zeng YM, Deng YY, Cheng JF. HOGG1 polymorphism in atrophic gastritis and gastric cancer after Helicobacter pylori eradication. World J Gastroenterol. 2010; 16:4476-4482.

27. Liu X, Xiao N, Guo W, Wu Y, Cai Z, He Q, Zhang L, Chen X, Sun C, Wang J, Zhu C, Jin H, Wang Y. The hOGG1 gene 5 '-UTR variant c.-53G $>C$ contributes to the risk of gastric cancer but not colorectal cancer in the Chinese population: the functional variation of hOGG1 for gastric cancer risk. J Cancer Res Clin Oncol. 2011; 137:1477-1485.

28. Engin AB, Karahalil B, Engin A, Karakaya AE. DNA repair enzyme polymorphisms and oxidative stress in a Turkish population with gastric carcinoma. Mol Biol Rep. 2011; 38:5379-5386.

29. Lu J, Yin Y, Du M, Ma G, Ge Y, Zhang Q, Chu H, Tong N, Wang M, Qiu J, Zhang Z. The association analysis of
hOGG1 genetic variants and gastric cancer risk in a Chinese population. Oncotarget. 2016; 7:66061-66068. doi: 10.18632/oncotarget.11802.

30. Hu WG, Pan RJ, Cai W, Wang ZT, Zhu ZG. Lack of association between the hOGG1 gene Ser326Cys polymorphism and gastric cancer risk: evidence from a case-control study and a meta-analysis. Genet Mol Res. 2015; 14:14670-14679.

31. Shinmura K, Kohno T, Kasai H, Koda K, Sugimura H, Yokota J. Infrequent mutations of the hOGG1 gene, that is involved in the excision of 8-hydroxyguanine in damaged DNA, in human gastric cancer. Jpn J Cancer Res. 1998; 89:825-828.

32. Ni M, Qiu J, He W, Wang X. The functional Ser326Cys polymorphism in hOGG1 is associated with gastric cancer risk: evidence from 1180 cases and 2444 controls. Eur J Gastroenterol Hepatol. 2012; 24:683-687.

33. Torre LA, Bray F, Siegel RL, Ferlay J, Lortet-Tieulent J, Jemal A. Global cancer statistics, 2012. CA Cancer J Clin. 2015; 65:87-108.

34. Oliveira C, Pinheiro H, Figueiredo J, Seruca R, Carneiro F. E-cadherin alterations in hereditary disorders with emphasis on hereditary diffuse gastric cancer. Prog Mol Biol Transl Sci. 2013; 116:337-359. 